\title{
Do barbed sutures with different surface textures have different effects on adhesion formation and histological features? An experimental blinded study in an animal model
}

\author{
Tolga Karacan ${ }^{1, A, D}$, Eser Ozyurek ${ }^{1, A}$, Lale Susan Türkgeldi ${ }^{2, C}$, Hüseyin Kıyak ${ }^{2, B, C}$, Simge Pesen ${ }^{1, B}$, Merve Yasti ${ }^{1}, C$, Taner Usta ${ }^{3, C}$ \\ ${ }^{1}$ Department of Obstetrics and Gynecology, University of Health Sciences, Bağcllar Training and Research Hospital, Istanbul, Turkey \\ ${ }^{2}$ Department of Obstetrics and Gynecology, University of Health Sciences, Kanuni Sultan Süleyman Training and Research Hospital, Istanbul, Turkey \\ ${ }^{3}$ Department of Obstetrics and Gynecology, Acıbadem University, Altunizade Hospital, Istanbul, Turkey \\ A - research concept and design; $B$ - collection and/or assembly of data; $C$ - data analysis and interpretation; \\ $D$ - writing the article; $E$ - critical revision of the article; $F$ - final approval of the article
}

\section{Address for correspondence}

Tolga Karacan

E-mail: tolgakaracan84@gmail.com

Funding sources

None declared

Conflict of interest

None declared

Received on January 3, 2018

Reviewed on March 13, 2018

Accepted on June 11, 2018

Published online on January 30, 2019

Cite as

Karacan T, Ozyurek E, Türkgeldi LS, et al. Do barbed sutures with different surface textures have different effects on adhesion formation and histological features? An experimental blinded study in an animal model. Adv Clin Exp Med. 2019;28(5):643-649. doi:10.17219/acem/92172

DOI

10.17219/acem/92172

Copyright

Copyright by Author(s)

This is an article distributed under the terms of the

Creative Commons Attribution Non-Commercial License

(http://creativecommons.org/licenses/by-nc-nd/4.0/)

\begin{abstract}
Background. The obstetrics and gynecology literature has expanded in recent years to include clinical trials assessing the use of barbed sutures. The difficulty of intracorporeal suturing continues to be a barrier to a wider use of laparoscopy. Although the use of barbed sutures has been shown to ease the process of laparoscopic suturing considerably, concerns have been raised regarding a potentially increased risk of adhesions or inflammation as a result of their use.
\end{abstract}

Objectives. The aim of this study was to determine whether differences in surface textures, resulting from the variations in the geometric configurations of barbs, lead to differences in intra-abdominal adhesion formation.

Material and methods. A total of 27 non-pregnant female Wistar Hannover rats, weighing 200-250 g, with intact uteri were used as an adhesion formation model. The rats were randomly assigned to 3 groups: barbed suture group 1, barbed suture group 2 and control group (no intracorporeal suture). A 2-centimeter vertical incision was performed on the anti-mesosalpingeal side of one of the uterine horns. The incision on the uterine horn was reapproximated with a running suture, entailing 3 needle punctures and left untied at one end. Six weeks after the operation, intra-abdominal adhesion formations were investigated both clinically and histopathologically.

Results. Clinical adhesion scores and histopathological parameters in both the barbed suture groups were statistically significantly higher than in the control group $(p<0.05)$. There was no significant difference between the barbed suture groups regarding the adhesion scores.

Conclusions. The 2 types of barbed sutures with different surface textures, used for myometrial closure, form a similar profile with respect to postoperative adhesion formation.

Key words: adhesion formation, barbed suture, knotless suture, myometrial closure, smooth suture 


\section{Introduction}

Since their first clinical use on human cadavers in 1967, barbed sutures have been employed in many areas, including general surgery, cosmetic surgery, orthopedic surgery, and gynecological surgery. At present, 3 types of barbed sutures present on the market are approved by the US Food and Drug Administration (FDA): Quill SRS bidirectional barbed sutures (Quill ${ }^{\mathrm{TM}}$ Self-Retaining System; Angiotech Pharmaceuticals, Vancouver, Canada), V-Loc unidirectional barbed sutures (V-Loc ${ }^{\mathrm{TM}}$ Absorbable Wound Closure Device; Covidien Ltd., Mansfield, USA) and Stratafix unidirectional and bidirectional barbed sutures (Stratafix ${ }^{\mathrm{TM}}$ Knotless Tissue Control Device; Ethicon Inc., Somerville, USA). ${ }^{1}$ These suture materials have sharp barbs that diffuse wound tension equally throughout the suture line by attaching tightly to each millimeter of tissue. ${ }^{2}$ Currently, the barbs on the surface of the sutures available on the market are placed on a monofilament thread in 3 different geometries and configurations., ${ }^{2,3}$

The obstetrics and gynecology literature has expanded in recent years to include clinical trials assessing the use of barbed sutures. Recent meta-analyses have shown that the use of barbed sutures, which ease intracorporeal suturing considerably during laparoscopic surgery, decreases the total duration of operations, the time spent on suturing and the estimated blood loss when compared with conventional sutures. ${ }^{4,5}$ Nevertheless, despite the many potential advantages of barbed sutures, some case reports have shown these new sutures to be associated with a higher risk of small bowel obstruction following myomectomy, vaginal cuff closure and sacrocolpopexy. Such adverse events might occur due to a potentially increased risk of adhesion formation or inflammation, resulting from the entrapment of barbs in the neighboring tissue. ${ }^{6-9}$ Adhesions can hamper the functions of abdominal organs, leading to infertility, chronic pelvic pain or increased morbidity. ${ }^{10}$

The aim of this study was to determine whether differences in surface textures, resulting from the variations in the geometric configurations of the barbs lead to differences in intra-abdominal adhesion formation. We compared 2 types of commercial barbed sutures with respect to clinical adhesion scores and histopathologic features in a rat animal model.

The study protocol was prepared in accordance with the National Institutes of Health (NIH) guidelines and approved by the Institutional Animal Care and Use Committee at the University of Health Sciences, Bağcılar Training and Research Hospital, Istanbul, Turkey (ethics committee registration No. 2016/111, dated September 28, 2016).

\section{Material and methods}

\section{Study animals}

A total of 27 non-pregnant female Wistar Hannover rats ( 9 rats allocated to each of the 3 groups), weighing 200-250 g, with intact uteri were used as an adhesion formation model. The rats were numbered sequentially and kept in standard plastic cages with sawdust-covered floors, in a room without windows, at a constant temperature of $21 \pm 1^{\circ} \mathrm{C}, 55-60 \%$ constant humidity, in a controlled environment with 12-hour cycles of darkness and light. The rats were given standard rat food and water ad libitum.

\section{Experimental groups}

The groups were assigned as follows:

- barbed suture group 1: animals sutured using Stratafix $^{\mathrm{TM}}$ Knotless Tissue Control Device, a synthetic absorbable monofilament suture with a single-angle barb design with 8 barbs/cm and 1 helix/5.08 mm (Fig. 1, A1);

- barbed suture group 2: animals sutured with V-Loc ${ }^{\mathrm{TM}}$ Absorbable Wound Closure Device, a synthetic absorbable monofilament suture with a dual-angle barb design with 20 barbs/cm and 1 helix/1.52 mm (Fig. 1, B1); and

- control group: no intracorporeal suturing.

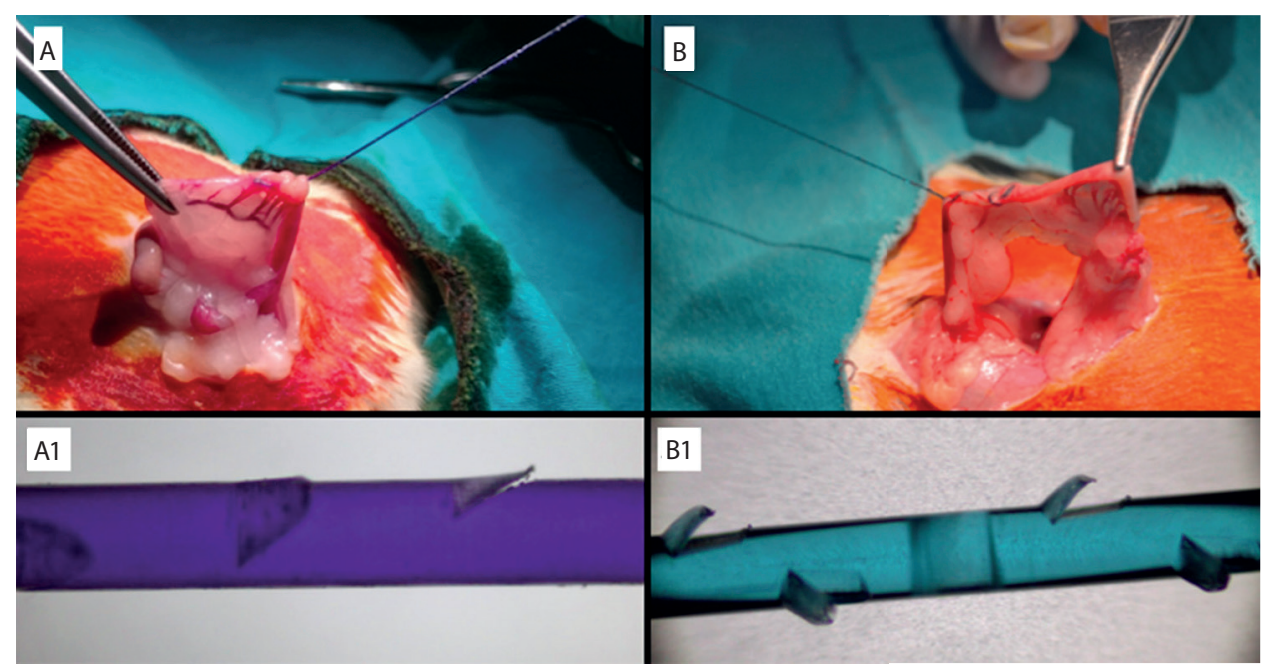

Fig. 1. Barbed suture group $1(A, A 1)$; barbed suture group 2 (B, B2).

A1 - A single-angle barb design with 8 barbs/cm and 1 helix/5.08 mm; B1 - A dual-angle barb design with $20 \mathrm{barbs} / \mathrm{cm}$ and 1 helix/1.52 $\mathrm{mm}$. 


\section{Surgical procedure}

All the rats were anesthetized with a single intramuscular injection of $50 \mathrm{mg} / \mathrm{kg}$ ketamine hydrochloride (Ketalar; Eczacıbaşı, Istanbul, Turkey) and $10 \mathrm{mg} / \mathrm{kg}$ xylazine (Rompun; Bayer Turk, Istanbul, Turkey). Each rat was assigned a number. The type of suture to be used and the uterine horn to be sutured were determined by assigning a closed envelope to each rat. Before surgery, the abdomen was shaved and antisepsis was obtained using a $10 \%$ povidoneiodine solution. Sterile powder-free surgical gloves were used during all procedures. The researcher (T.K.) made a 5-centimeter abdominal midline vertical incision to expose the uterine horns.

The animal model to test for intraperitoneal adhesions has previously been used by Api et al. ${ }^{9}$ In the control group, the abdominal cavities were entered as previously described and closed without performing any further surgical procedures. In the study groups, a 2 -centimeter incision was made with a No. 15 scalpel on the anti-mesosalpingeal side of one of the uterine horns to mimic a myomectomy wound. The incision on the uterine horn was reapproximated with a running suture, entailing 3 needle punctures and left untied at one end. The free end of the barbed suture was cut at the same level as the tissue, without leaving any protruding parts. No adhesion barriers were left in the peritoneal cavity. All the uterine horn procedures were performed by the same researcher (T.K.). Blood and fibrin were removed by rinsing the surgical site with a physiological serum solution after confirmation of hemostasis, and the abdominal wall was closed with simple interrupted sutures using 3-0 polyglactin 910 sutures (Vicryl ${ }^{\circledR}$; Ethicon Inc.). The operation times were limited to about 10 min for each rat to prevent drying of the tissue with room air. After the operations, the rats were housed separately.

\section{Macroscopic examination}

After the completion of the 6-week recovery period, all the animals were sacrificed using pentobarbital $250 \mathrm{mg} / \mathrm{kg}$, and second-look laparotomies were performed. During the second-look operation, the researchers examined all the possible locations for intra-abdominal adhesions under the laparotomy incision, between the intestinal loops, at the uterine horns, etc. - by careful manipulation with a fine tissue holder. Visible intra-abdominal adhesions were identified and scored according to the adhesion scoring system devised by Leach et al., ${ }^{11}$ by researchers blinded to the previous allocation of the suture type in each animal (S.P. and E.O.).

The extent of adhesions involving the uterus was scored as follows: 0 = no uterine adhesions; $1: 1-25 \%$ involvement; 2: $26-50 \%$ involvement; 3 : $51-75 \%$ involvement; and 4:
76-100\% involvement. Adhesions were further scored during gross examination to determine the severity as follows: 0: no adhesion; 1 : filmy avascular adhesions; 2 : vascular or opaque adhesions; and 3: a cohesive attachment of the uterine horns to each other or to other abdominal organs. The degree of adhesion formation was evaluated with the following adhesion scores: 0: no adhesion; 1 : the adhesion could be separated from tissue with gentle traction; 2 : the adhesion could be separated from tissue with moderate traction; and 3: separation required a sharp dissection. The sum of the 3 parameters was used as the total score for each group (Fig. 2).

\section{Histopathological examination}

After completing the scoring, the adhesions were also examined histopathologically (by 2 histopathologists blinded to the groups), and graded for the presence of inflammation and fibrosis, using grading scales previously published by Hooker et al. ${ }^{12}$ For this purpose, the uterine horns sutured with barbed sutures were excised, and then fixed in a $10 \%$ buffered formalin solution for $24 \mathrm{~h}$. After fixation, routine tissue-processing procedures were performed and the sampled tissues were embedded in paraffin. The paraffin wax blocks were sectioned $(4 \mu \mathrm{m})$ using a microtome (Leica RM 2125RTS; Leica Biosystems, Nussloch, Germany). The prepared sections were stained with hematoxylin-eosin (H\&E) stain for inflammatory cell reaction and with Masson's trichrome stain for collagen fibers. An experienced histopathologist blinded to which uterine horn had been sutured examined the tissue sections under a light microscope (Olympus BX51; Olympus Corp., Tokyo, Japan). Inflammation was scored as follows: 0: no inflammation; 1: presence of giant cells, occasional lymphocytes and plasma cells; 2 : presence of giant cells, plasma cells, eosinophils, and neutrophils; and 3: presence of many inflammatory cells and microabscesses. The amount of fibrosis was scored as: 0: no fibrosis; 1: minimal, loose fibrosis; 2: moderate fibrosis; and 3: florid, dense fibrosis.

\section{Statistical analysis}

Descriptive and comparative statistical analyses were conducted using the Statistical Package for Social Sciences (SPSS) v. 19.0 (SPSS Inc., Chicago, USA). The results were expressed as median, mean \pm standard error of the mean (SEM) and 95\% confidence interval (95\% CI). The Kruskal-Wallis test and Wilcoxon rank-sum test were used to compare the differences in scores among the groups, and the Bonferroni adjustment was used. For inter-rater reliability, the results of the observations scored by S.P. and E.O. were statistically analyzed by Cohen's kappa test, and a k-value of $80 \%$ was considered the minimum acceptable inter-rater agreement. 


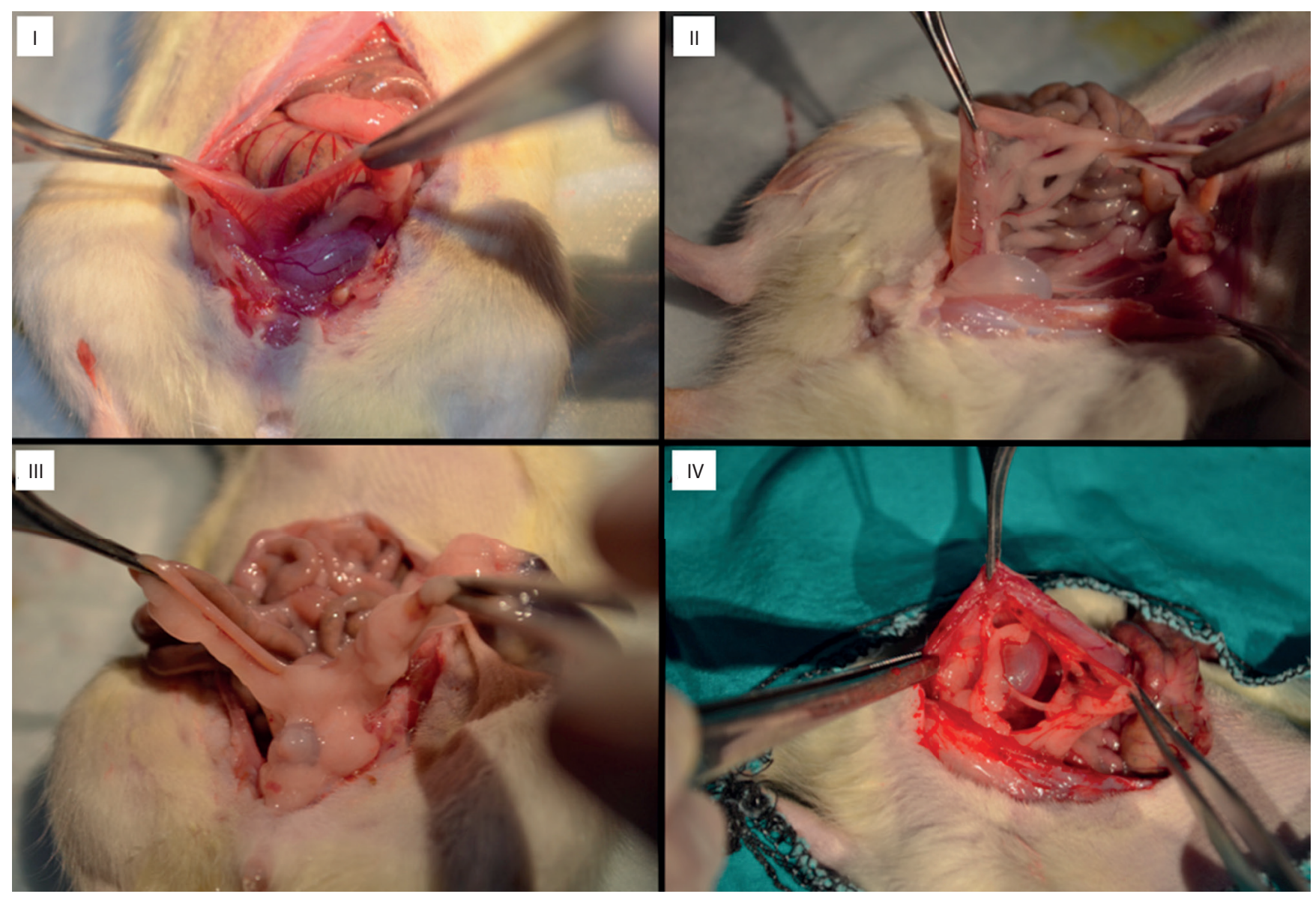

Fig. 2. Macroscopic view of rat uterine horns with various degrees of adhesion formation during the second-look laparotomy: I - the uterine horns without adhesion formation; II - filmy adhesion on the right horn; III - dense adhesion between the uterine horns; IV - a very dense attachment of uterine horns to each other or to other abdominal organs

\section{Results}

The surgical procedures on all 27 animals were performed successfully without any complications. All laparotomy areas were intact and no deaths occurred. The histopathological parameters (collagen deposition and inflammatory cell reaction) and clinical adhesion scores (extent, severity, degree, and total adhesion scores) of the groups are summarized in Table 1. Statistically significant differences were present between all the 3 groups in both clinical adhesion scores and histopathological parameters. A subgroup analysis showed that clinical adhesion scores and histopathological parameters in both barbed suture groups were statistically significantly higher than in the control group ( $\mathrm{p}<0.05)$. The inflammatory cell response and collagen deposition were higher in barbed suture group 2 than in barbed suture group 1; however, this difference was not statistically significant ( $\mathrm{p}>0.05$ ) (Fig. 3). Similarly, there was no significant difference between the barbed suture groups in their adhesion scores ( $\mathrm{p}>0.05)$ (Fig. 4).

Table 1. Comparison of clinical and histological adhesion scores among the 3 groups

\begin{tabular}{|l|c|c|c|c|c|}
\hline \multicolumn{1}{|c|}{ Parameter } & Barbed suture group 1 & \multicolumn{1}{|c|}{ Barbed suture group 2 } & Control group \\
\hline Extent score & $3(2.55 \pm 0.33 ; 1.91-3.19)$ & $3(2.60 \pm 0.23 ; 2.15-3.05)$ & $1(1.11 \pm 0.30 ; 0.53-1.69)$ \\
\hline Severity score & $2(1.77 \pm 0.22 ; 1.34-2.20)$ & $2(1.88 \pm 0.26 ; 1.38-2.38)$ & $1(0.75 \pm 0.23 ; 0.30-1.20)$ \\
\hline Degree score & $2(1.88 \pm 0.35 ; 1.20-2.56)$ & $2(1.77 \pm 0.27 ; 1.25-2.29)$ & $0(0.55 \pm 0.24 ; 0.08-1.02)$ \\
\hline Total score & $6(6.22 \pm 0.32 ; 5.60-6.84)$ & $6(6.33 \pm 0.37 ; 5.61-7.05)$ & $2(2.44 \pm 0.60 ; 1.27-3.61)$ \\
\hline & \multicolumn{2}{|c|}{ Histological features } & $<0.01$ \\
\hline Collagen deposition & $2(1.88 \pm 0.20 ; 1.49-2.27)$ & $2(2.00 \pm 0.23 ; 1.55-2.45)$ & $0(0.44 \pm 0.17 ; 0.11-0.77)$ \\
\hline Inflammatory cell reaction & $2(2.11 \pm 0.26 ; 1.61-2.61)$ & $2(2.22 \pm 0.22 ; 1.79-2.65)$ & $1(0.77 \pm 0.22 ; 0.34-1.20)$ \\
\hline
\end{tabular}

* Values are presented as median (mean \pm standard error of the mean (SEM), $5^{\text {th }}-95^{\text {th }}$ percentile, $95 \%$ confidence interval $(95 \%$ CI)). 

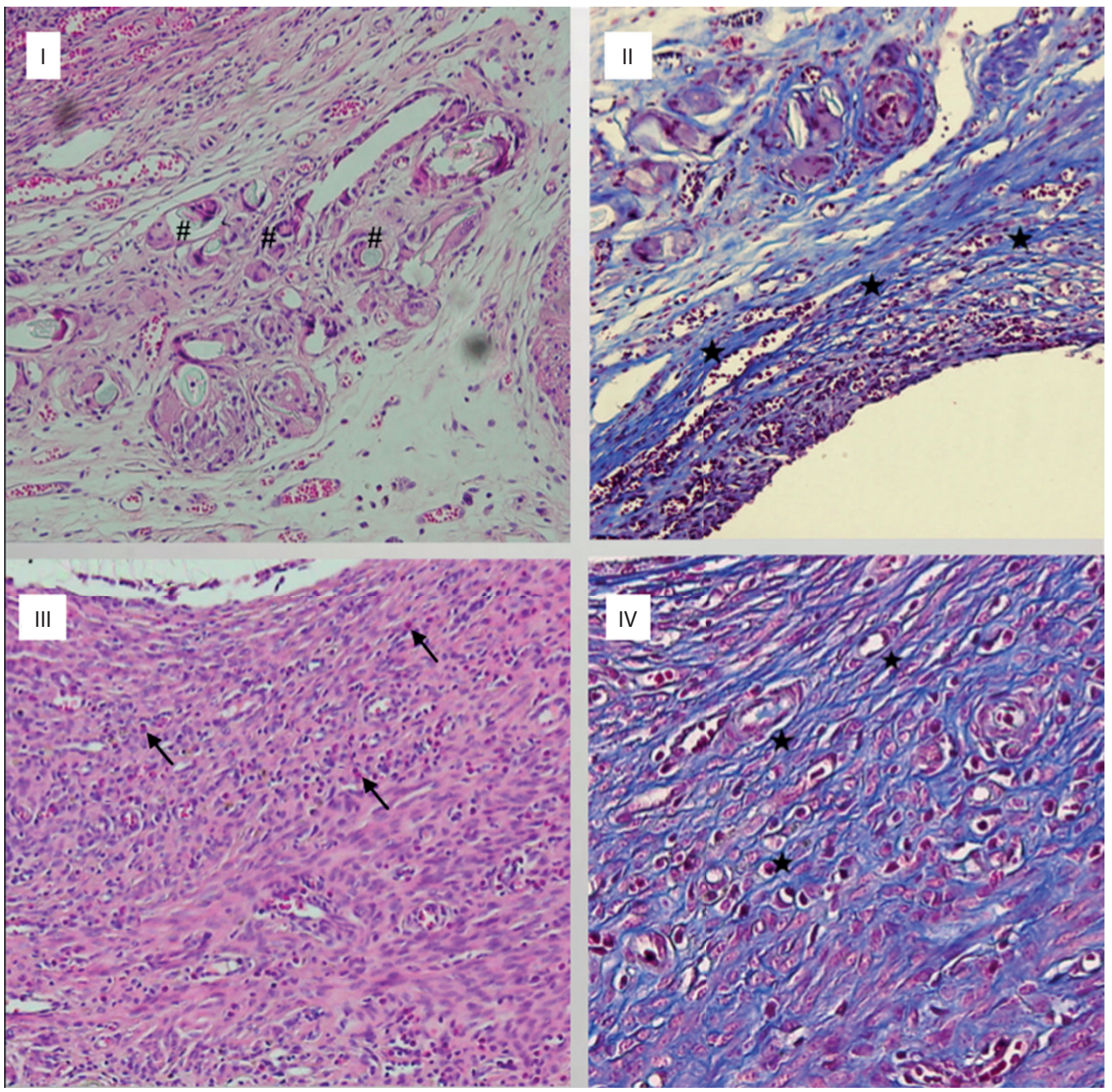

Fig. 3. Representative tissue specimens of hematoxylin-eosin (H\&E) staining (I, III) and Masson's trichrome staining (II, IV) (original magnification $\times 20$ and $\times 40$ ); I, II - barbed suture group 1; III, IV - barbed suture group 2

$\rightarrow$ polymorphonuclear leukocytes; \# - foreign-body type giant cell; * - collagen deposition.

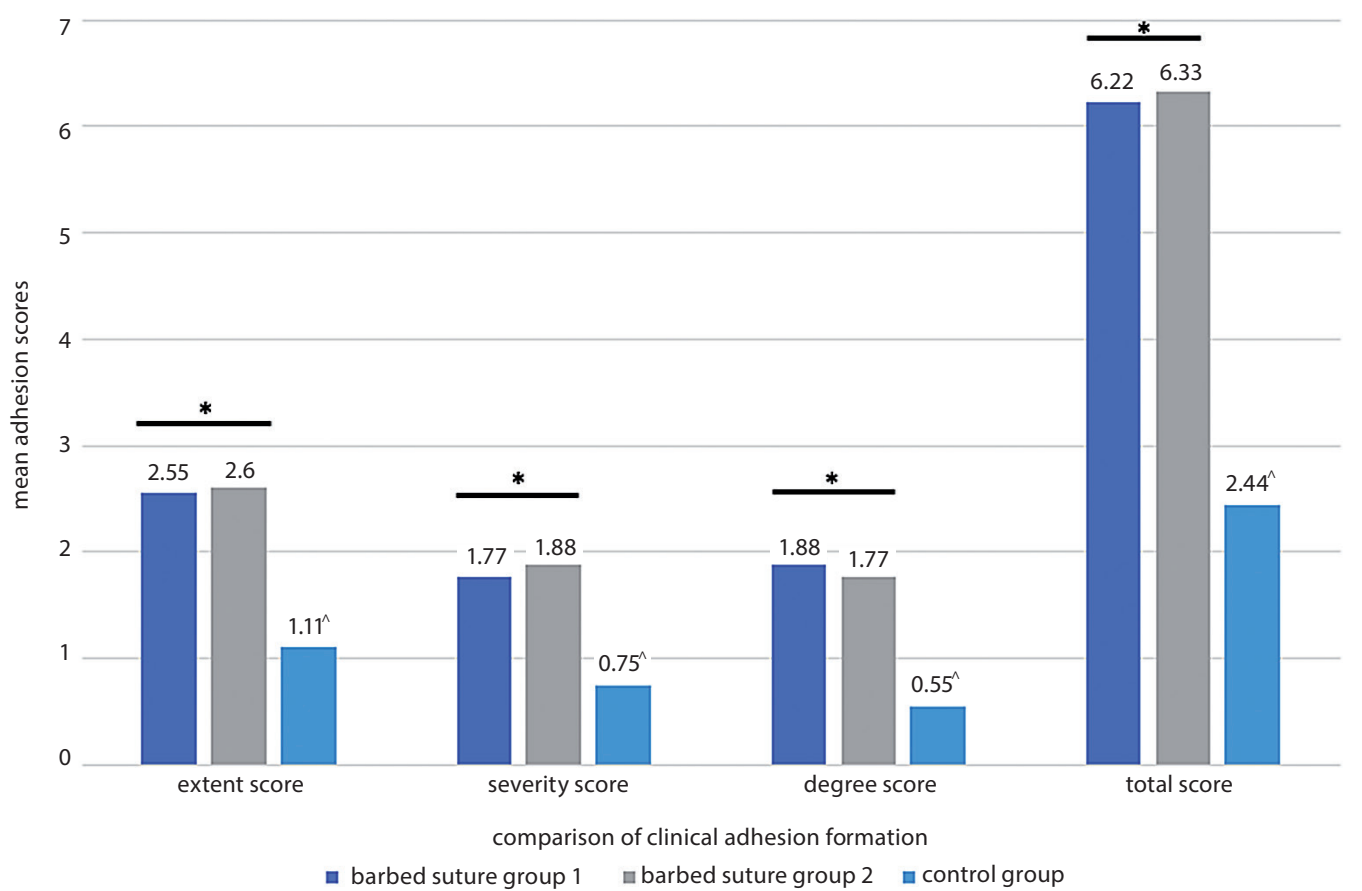

Fig. 4. Comparison of mean the clinical adhesion scores among the 3 groups during the second-look operation, 6 weeks after the first operation in a myomectomy wound model of 27 rats

* no significant difference between the barbed suture groups in clinical adhesion scores $(p>0.05) ;{ }^{\wedge}$ clinical adhesion scores in both barbed suture groups were statistically significantly higher than in the control group $(p<0.05)$.

The inter-rater reliability values for clinical adhesion scores were 0.80 kappa for extent, 0.94 kappa for severity and 0.84 kappa for degree $(\mathrm{p}<0.001)$. Inter-rater reliability for histopathological parameters were 0.94 kappa for collagen deposition and 0.89 kappa for inflammatory cell reaction. 


\section{Discussion}

In this study, the effects of different surface textures of barbed sutures on adhesion formation in a rat uterine horn model were investigated both clinically and histopathologically. The results of the present study demonstrated that different surface textures of barbed sutures and various configurations of barbs cause adhesion formation of a comparable extent, severity and degree.

The difficulty of intracorporeal suturing continues to be a barrier to a wider use of laparoscopy. Although the use of barbed sutures has been shown to ease the process of laparoscopic suturing considerably, concerns have been raised regarding a potentially increased risk of adhesions or inflammation as a result of their use. ${ }^{13}$ Adhesions can cause loss of function of abdominal organs and lead to infertility, chronic pelvic pain, bowel obstruction, and significant morbidity. ${ }^{10}$ Suture materials can act as foreign bodies within the abdomen, provoking inflammatory reactions that lead to peritoneal adhesion formation. Contrary to the chronic process of adhesion formation, small bowel obstruction can occur acutely and may be related to the anchoring of sharp barbs onto the neighboring tissue rather than to the presence of a foreign material within the abdomen. Many previous studies have compared the effects of different suture characteristics on adhesion formation. ${ }^{14,15}$ However, to our knowledge, the relative outcome differences between the 2 types of barbed sutures with different barb geometries, barb numbers and helicity have not previously been studied.

In 2 studies conducted by Einarsson et al., a bidirectional polydioxanone barbed suture (Quill) and a polyglactin 210 standard suture (Vicryl) were compared with respect to the amount of adhesion formation. ${ }^{15,16}$ According to the results of those studies, while the adhesion percentage was $52.2 \%$ and the median adhesion score was $4.0(0-10)$ for cases in which barbed sutures were used, the same parameters for cases in which standard sutures were used were $43 \%$ and 0 (0-9), respectively. Barbed sutures were found to induce a similar degree of inflammatory reaction as compared to standard sutures; however, their adhesion scores were $10 \%$ higher than those of standard sutures. Nevertheless, this $10 \%$ difference in adhesion scores between the 2 types of sutures did not reach statistical significance. In a study by Api et al., V-loc barbed sutures were found to provoke less inflammatory tissue response, but a statistically significantly higher amount of clinical adhesion formation than polyglactin 910 (Vicryl) sutures. ${ }^{9}$ Similar results were observed when V-loc and Vicryl sutures were compared for cystotomy repair in rats. ${ }^{17}$ Different results in the adhesion scores reported by Einarsson et al. and Api et al. when barbed sutures (Quill and V-loc) were compared with standard sutures (Vicryl) may not only be a result of the different methodologies used in the studies (for example, the type of animals used - rat vs ewe, the type of instrument used for the incision over the horns
- scalpel vs harmonic scalpel, or the follow-up period after the surgeries -6 weeks vs 3 months), but also of the different surface textures of the sutures. ${ }^{9,14,15}$ These histological features and the differences in the degree of adhesions may be explained by the mechanical effect of the barbed suture material on intra-abdominal peritoneal surfaces. Surface textures of barbed sutures may cause the entanglement of adjacent tissues by creating a rough environment on the peritoneal surface, which may consequently produce adhesions. ${ }^{9}$ The 2 barbed suture brands with different surface textures that were used in the present study were not found to cause a difference either in clinical adhesion scores or in histological parameters.

Researchers have attributed the different biometric behavior patterns between various barbed sutures to differences in the surface designs and diameters of sutures. In a similar study with a similar intent, Jordan et al. aimed to determine whether 2 different barbed sutures behaved differently biomechanically ex vivo. ${ }^{3}$ With this purpose, they compared barbed sutures on pig flexor tendons with respect to the maximum load (ultimate tensile strength) and displacement. V-loc was found to have similar displacement patterns as Stratafix, but it had a higher tensile strength. Similar results were reported in studies in the field of esthetic surgery. ${ }^{14}$

The present study has some noteworthy strengths. The methodology used to assess and compare the risk of adhesion formation connected with using barbed sutures is different compared to previous studies, in which subjects were used as their own controls; in our study, the study groups and the control group comprised different subjects. This is a simple but significant methodological difference, because the adhesion conditions measured in each subject may not necessarily be the adhesion potential rendered by the suture material at one circumscribed location of the whole abdominal environment (especially with barbed sutures, which are hypothesized to form a rough peritoneal surface entangling the neighboring structures to cause adhesions); hence, in principle, we believe the subjects should not serve as their own controls. The other strength of the present study was that 2 researchers blinded to the study groups assessed the degree of adhesion formation intraoperatively. Consequently, underestimation or overestimation of the degree of adhesions as a result of photographic evaluation of specimens postoperatively were prevented.

However, some limitations inherent to animal studies are also present in this study. Most importantly, it is not certain to what extent the results of this study are transferrable to human subjects. Myomectomy was not performed in our rat model; instead, an imitation of it was performed by making an incision over one of the uterine horns. It may not be possible to determine to what extent a simple incision and closure may evoke an adhesion response similar to that of a myomectomy. Certainly, human studies will be required to retest our results.

In our study, the control group served as a sham group to distinguish the effects of laparotomy and anesthesia. 


\section{Conclusions}

To date, there is no final consensus on the ideal suture material to be used for myometrial closure. An ideal suture material would provide adequate tensile strength during the healing process, cause minimal foreign-body reaction and would be easy to use. The current clinical literature has shown the performance of absorbable barbed sutures to be equivalent to conventional absorbable smooth sutures for soft tissue reapproximation in gynecology. ${ }^{18,19}$ Future studies are necessary to determine the association between different types of barbed sutures and adhesion formation in humans and to evaluate the long-term tensile strength in myometrial defects repaired with different types of barbed sutures.

In conclusion, the results of the present study indicate that these 2 types of barbed sutures with different surface textures have a similar profile regarding postoperative adhesion formation when used for myometrial closure.

\section{References}

1. Greenberg JA. The use of barbed sutures in obstetrics and gynecology. Rev Obstet Gynecol. 2010;3(3):82-91.

2. Greenberg JA, Goldman RH. Barbed suture: A review of the technology and clinical uses in obstetrics and gynecology. Rev Obstet Gynecol. 2013;6(3-4):107-115.

3. Jordan MC, Hölscher-Doht S, Jakubietz MG, Jakubietz G, Meffert RH, Schmidt K. Suture material for flexor tendon repair: 3-0 V-Loc versus 3-0 Stratafix in a biomechanical comparison ex vivo. J Orthop Surg Res. 2014:9:72.

4. Tulandi T, Einarsson Jl. The use of barbed suture for laparoscopic hysterectomy and myomectomy: A systematic review and meta-analysis. J Minim Invasive Gynecol. 2014;21(2):210-216.

5. Greenberg JA, Einarsson JI. The use of bidirectional barbed suture in laparoscopic myomectomy and total laparoscopic hysterectomy. J Minim Invasive Gynecol. 2008;15(5):621-623.

6. Burchett MA, Mattar SG, McKenna DT. latrogenic intestinal and mesenteric injuries with small bowel volvulus following use of barbed suture during laparoscopic myomectomy. J Laparoendosc Adv Surg Tech A. 2013;23(7):632-634.
7. Thubert T, Pourcher G, Deffieux X. Small bowel volvulus following peritoneal closure using absorbable knotless device during laparoscopic sacral colpopexy. Int Urogynecol J. 2011;22(6):761-763.

8. Donnellan NM, Mansuria SM. Small bowel obstruction resulting from laparoscopic vaginal cuff closure with a barbed suture. J Minim Invasive Gynecol. 2011;18(4):528-530.

9. Api M, Boza A, Cikman MS, Aker FV, Onenerk M. Comparison of barbed and conventional sutures in adhesion formation and histological features in a rat myomectomy model: Randomized single blind controlled trial. Eur J Obstet Gynecol Reprod Biol. 2015;185:121-125.

10. Neff MR, Holtz GL, Betsill WL. Adhesion formation and histologic reaction with polydioxanone and polyglactin suture. Am J Obstet Gynecol. 1985;151(1):20-23.

11. Leach RE, Burns JW, Dawe EJ, SmithBarbour MD, Diamond MP. Reduction of postsurgical adhesion formation in the rabbit uterine horn model with use of hyaluronate/carboxymethylcellulose gel. Fertil Steril. 1998;69(3):415-418.

12. Hooker GD, Taylor BM, Driman DK. Prevention of adhesion formation with use of sodium hyaluronate-based bioresorbable membrane in a rat model of ventral hernia repair with polypropylene mesh: A randomized, controlled study. Surgery. 1999;125(2):211-216.

13. Api M, Cikman MS, Boza A, Rabus MB, Onenerk M, Aker FV. Peritoneal closure over barbed suture to prevent adhesions: A randomized controlled trial in an animal model. J Minim Invasive Gynecol. 2015; 22(4):619-625.

14. Zaruby J, Gingras K, Taylor J, Maul D. An in vivo comparison of barbed suture devices and conventional monofilament sutures for cosmetic skin closure: Biomechanical wound strength and histology. Aesthetic Surg J. 2011;31(2):232-240.

15. Einarsson Jl, Vonnahme KA, Sandberg EM, Grazul-Bilska AT. Barbed compared with standard suture: Effects on cellular composition and proliferation of the healing wound in the ovine uterus. Acta Obstet Gynecol Scand. 2012;91(5):613-619.

16. Einarsson Jl, Grazul-Bilska AT, Vonnahme KA. Barbed vs standard suture: Randomized single-blinded comparison of adhesion formation and ease of use in an animal model. J Minim Invasive Gynecol. 2011;18(6):716-719.

17. Petrut $B$, Hogea M, Fetica B, et al. In-vivo assessment of barbed suturing thread with regard to tissue reaction and material absorption in a rat model. Clujul Med. 2013;86(4):371-376.

18. Dennis C, Sethu S, Nayak S, Mohan L, Morsi Y, Manivasagam G. Suture materials: Current and emerging trends. J Biomed Mater Res. 2016; 104(6):1544-1549.

19. Greenberg JA, Clark RM. Advances in suture material for obstetric and gynecologic surgery. Rev Obstet Gynecol. 2009;2(3):146-158. 